\title{
Numerical Solution for the 2D Linear Fredholm Functional Integral Equations
}

\author{
Neda Khaksari $\mathbb{D}^{1},{ }^{1}$ Mahmoud Paripour ${ }^{(D},{ }^{2}$ and Nasrin Karamikabir ${ }^{1}{ }^{1}$ \\ ${ }^{1}$ Department of Mathematics, Hamedan Branch, Islamic Azad University, Hamedan, Iran \\ ${ }^{2}$ Department of Computer Engineering and Information Technology, Hamedan University of Technology, Hamedan, Iran
}

Correspondence should be addressed to Mahmoud Paripour; m_paripour@yahoo.com

Received 14 August 2021; Accepted 19 October 2021; Published 10 November 2021

Academic Editor: Xian-Ming Gu

Copyright ( $\odot 2021$ Neda Khaksari et al. This is an open access article distributed under the Creative Commons Attribution License, which permits unrestricted use, distribution, and reproduction in any medium, provided the original work is properly cited.

In this work, a numerical method is applied for obtaining numerical solutions of Fredholm two-dimensional functional linear integral equations based on the radial basis function (RBF). To find the approximate solutions of these types of equations, first, we approximate the unknown function as a finite series in terms of basic functions. Then, by using the proposed method, we give a formula for determining the unknown function. Using this formula, we obtain a numerical method for solving Fredholm twodimensional functional linear integral equations. Using the proposed method, we get a system of linear algebraic equations which are solved by an iteration method. In the end, the accuracy and applicability of the proposed method are shown through some numerical applications.

\section{Introduction}

The integral equations technique is broadly applied for solving several equations in physics, mathematics, and engineering $[1,2]$. There are many studies about integral problems which deal with the analysis of these types of equations and solution. Many equations in physics and engineering may be modeled applying second kind twodimensional integral equations. Fredholm integral equations have utilizations in plasma physics, electrical engineering [3], and telegraph equations [4]. Due to the limited area of closed form solutions, efforts are required to make accurate numerical methods and efficient algorithms for simulating such models. A new interpolation method for functional integral equation is introduced by Rashed [5] and then developed in more detail by other researchers. Among different approaches, the RBF method is one of the best numerical methods for approximation of the solutions. In this study, RBF is used to approximate the solution of the Fredholm linear functional two-dimensional integral equation of second kind as follows:

$$
l(X)=u(X)+A(X) u(h(X))+\lambda \int_{a}^{b} \int_{c}^{d} K(X, T) u(T) \mathrm{d} T,
$$

where $A(X), h(X)$, and $l(X)$ are the analytical known continuous functions, and the kernel $K(X, T)$ is a function in $[a, b] \times[c, d]$. In the above equation, $T=\left[t_{1}, t_{2}\right]$, and $X=\left[x_{1}, x_{2}\right]$. In order to approximate function $u(X)$, RBF interpolation is used in distinct points from the domain definition. To do so, the following linear combination is considered:

$$
u(X) \approx \sum_{i=0}^{n} \alpha_{i} \phi_{i}(X)
$$

where $\phi_{i}(X)$ can be one of the basis functions in Table 1. One of the most important bases is the radial basis, and the corresponding method for these functions is radial basis function. The RBF method is an effective method for the interpolation of scattered data. Also, we use the radial basis functions in this study to get good results at reasonably low computational cost. The advantage of the proposed method consists mainly in the fact that it is easy to use and implement but shows good approximations of the solution at a 
TABLE 1: Well-known functions generating RBF.

\begin{tabular}{lc}
\hline Function & Definition \\
\hline Gaussian (GA) & $\phi(r)=\exp \left(-\sigma r^{2}\right)$ \\
Multiquadric (MQ) & $\phi(r)=\sqrt{r^{2}+\sigma^{2}}$ \\
Inverse multiquadric (IMQ) & $\phi(r)=\left(r^{2}+\sigma^{2}\right)^{(-(1 / 2))}$ \\
Inverse quadric (IQ) & $\phi(r)=\left(r^{2}+\sigma^{2}\right)^{(-1)}$ \\
\hline
\end{tabular}

given set of nodes. Furthermore, there is another catalog of used studies $[6,7]$ that study the solution of problems with radial basis functions in the interior of the domain. In the RBF set, $\left\{\phi_{i}\right\}_{i=1}^{n}$ is shown as follows:

$$
\phi_{i}: \mathbb{R}^{n} \longrightarrow \mathbb{R}, \quad \phi_{i}(\alpha)=\phi\left(\left\|x-x_{i}\right\|\right),
$$

where $\|\cdot\|$ is the Euclidean norm and $x_{i}$ is the center of RBF. One of the famous functions which produces RBF is the Gaussian function, and more functions are presented in Table 1 . These functions according to having the parameter $\alpha_{i}$ have exponential convergence [8].

$\mathrm{RBF}$ is a function with a quantity real positive, and its values depend on distance from center. The choice of the center point can have an arbitrary distribution; therefore, scattered data can be used for the necessary calculation. Interpolation by the RBF method has many benefits including high accuracy and applicable in scattered data and irregular domains. Also, there is appearance of existence and unique result, while there are little restrictions on dimension. Another important advantage of RBF is that it does not need triangulation from the data points, unlike other numerical methods such as multivariable polynomial interpolation or splines [9]. The concept of radial basis function was first introduced by Hardy [10, 11]. Franke [12], in his study, compared interpolation methods and made RBF more famous. Buhmann and Micchelli $[13,14]$ proved that the multiquadric interpolation advanced and application of RBF. Kansa $[15,16]$ used the multiquadrics method for estimating partial derivative and partial differential equations. Since then, a number of researchers have extensively used approaches based on RBF in many articles [17-22]. Now, to approximate the target function, $\phi_{i}(X)$ is substituted for one of the bases functions. As an example, consider Gaussian function, and then, we would have

$$
\phi_{i}(X)=e^{-\sigma\left\|X-X_{i}\right\|^{2}} .
$$

Similarly,

$$
u(h(X)) \approx \sum_{i=0}^{n} \alpha_{i} \phi_{i}(h(X)),
$$

is established when $h$ is a known function. This study is organized as follows. Section 2 presents the RBF method to approximate Fredholm two-dimensional integral equations. Section 3 presents some examples to analyze the proposed approach and show its efficiency. Finally, we will express the conclusion.

\section{Methodology}

In the $n$-dimensional Euclidean space, take $m$ distinct point into consideration $\left(x_{1}, x_{2}, \ldots, x_{m}\right) \in \mathbb{R}^{n}$. In this space, the approximation function known as $\left(l\left(x_{1}\right), l\left(x_{2}\right), \ldots, l\left(x_{n}\right)\right)$ are the value of the given points. Let us consider the following continuous functions $w: \mathbb{R}^{n} \longrightarrow R$, so that $w\left(x_{j}\right)=$ $l_{j}$ for $j=1,2, \ldots, m$. RBF is based on a continuous function, so that $\phi: R^{+} \longrightarrow R^{+}$and $\|\cdot\|$ is a norm in $\mathbb{R}^{n}$. Therefore, we can have $w$ in the following form:

$$
w(x)=\sum_{j=1}^{m} \alpha_{j} \phi\left(\left\|x-x_{j}\right\|\right),
$$

where $\alpha_{j}$ are the scalar parameters which have to be determined. So, $w$ approximates $l$ at $x_{j}$ point for $j=1,2, \ldots, m$. Now, due to interpolation conditions, a linear system is defined as

$$
\Delta \alpha=L,
$$

where $L=\left(l_{1}, l_{2}, \ldots, l_{n}\right)^{T}, \alpha=\left(\alpha_{1}, \alpha_{2}, \ldots, \alpha_{n}\right)^{T}$, and $\Delta \epsilon$ $\mathbb{R}^{n \times n}$ is called the interpolation matrix and is shown as

$$
\Delta=\left[A_{i j}\right]_{n \times n},
$$

where $A_{i j}=\phi\left(\left\|x_{i}-x_{j}\right\|\right)$. The interpolation matrix is nonsingular because it is a positive definite matrix that definition of the positive definite matrix is given in [11]; therefore, there is unique and existence for coefficients $\alpha_{j}$.

2.1. The Convergence of RBF Approach. To investigate the convergence of the RBF approach, suppose that the data points are on equispaced grids in $\mathbb{R}^{n}$. The distance is indicated by $h$, when $h \longrightarrow 0$. In fact, there are infinite uniform grids from $h$. Considering $w$ as an approximation of function $l$ by using RBF, the uniform difference between $w$ and $l$ with the same rate is same power of $h$ toward zero. In this article, the convergence of Gaussian function is examined. Although, the same approach is applicable for other basis functions given in Table 1 .

Definition 1 (Native space [23]). The native space that is conditionally positive definite on $\Omega$ with respect to the p-unisolvent subset is defined by

$$
N_{\phi(\Omega)}:=R\left(F_{\phi(\Omega)}\right)+p \text {, }
$$

where $\quad R: F_{\phi(\Omega)} \longrightarrow C(\Omega), R(f)(x)=(f, G(\cdot, x))_{\phi}$, and $G(\cdot, x)$ is given in [23]. The space is equipped with a semiinner product via

$$
(f, g)_{N_{\phi(\Omega)}}=\left(R^{-1}\left(f-T_{p} f\right), R^{-1}\left(g-T_{p} g\right)\right) \phi,
$$

where $T_{p}: C(\Omega) \longrightarrow p$ and $T_{p}(f)=\sum_{k=1}^{\mathbb{Q}} f(\xi K) p_{k}$.

The concept of convergence for RBF interpolation in the domain $\Omega \in \mathbb{R}^{n}$ is based on functions over native spaces $N_{\phi(\Omega)}$. For same radial basis functions such as Gaussian, 
which are strictly positive definite basis functions, we can make the native space from positive conditional basis functions as the completion of the pre-Hilbert spaces. Take the following linear space into consideration:

$$
F_{\phi(\Omega)}=\left\{\sum_{j=1}^{N} \alpha_{j} \phi\left(\left\|\cdot-x_{j}\right\|\right), \quad \alpha \in \mathbb{R}^{N}, x_{j} \in \Omega, j=1, \ldots, N\right\} .
$$
space:

Definition of inner product $F_{\phi(\Omega)}$ becomes a pre-Hilbert

$$
\begin{aligned}
& \left(\sum_{j=1}^{N} \alpha_{j} \phi\left(\left\|\cdot-x_{j}\right\|\right), \sum_{k=1}^{N} \beta_{k} \phi\left(\left\|\cdot-x_{k}\right\|\right)\right)_{\phi(\Omega)} \\
& :=\left(\sum_{j=1}^{N} \sum_{k=1}^{N} \alpha_{j} \beta_{k} \phi\left(\left\|x_{j}-x_{k}\right\|\right)\right) .
\end{aligned}
$$

By applying RBF, the fill distance from $h$ is considered as follows:

$$
h_{N}:=\sup \min _{x \in \Omega}\left\|x-x_{j}\right\|_{2}, \quad j=1,2, \ldots, N .
$$

So, for sufficiently small $h_{N}$ and data $x_{j}$, the following relations have resulted for Gaussian:

$$
\left\|l-w_{f, N}\right\|_{L_{\infty}(\Omega)} \leq e^{\left(-\alpha \log h_{N} / h_{N}\right)}\|f\|_{N_{\phi(\Omega)}} .
$$

The concept of convergence for RBF approximation is defined for functions in the domain $\Omega \in \mathbb{R}^{N}$ that are on native spaces $N_{\phi(\Omega)}$. Convergence analysis can be proved by a similar process in [24] for this method.

\subsection{Application of RBF with Fredholm Linear Functional Two-}

Dimensional Integral Equation. In this section, we interpolate a continuous function $u: \mathbb{R}^{n} \longrightarrow \mathbb{R}$ on a set $X=$ $\left\{x_{1}, \ldots, x_{n}\right\}$ with choosing the radial basis function for $\phi: \mathbb{R}^{n} \longrightarrow \mathbb{R}$ that is radial in the sense. Now, we use the RBF interpolation for approximation $u(X)$. In the introduction, we remember that Fredholm two-dimensional integral equation of second kind was defined as follows:

$$
l(X)=u(X)+A(X) u(h(X))+\lambda \int_{a}^{b} \int_{c}^{d} K(X, T) u(T) \mathrm{d} T,
$$

where $T=\left(t_{1}, t_{2}\right), X=\left(x_{1}, x_{2}\right)$. The RBF interpolation is used to approximate the above integral equation. In order, the linear component of function $\phi_{i}$ is substituted in $u(X)$ as the following form:

$$
u(X) \approx \sum_{i=0}^{n} \alpha_{i} \phi_{i}(X)
$$

and

$$
u(h(X)) \approx \sum_{i=0}^{n} \alpha_{i} \phi_{i}(h(X))
$$

where $h:[a, b] \times[c, d] \longrightarrow[a, b] \times[c, d]$ is a known function. We would also have

$$
\begin{aligned}
\int_{a}^{b} \int_{c}^{d} K(X, T) u(T) \mathrm{d} T & \approx \int_{a}^{b} \int_{c}^{d} K(X, T) \sum_{i=0}^{n} \alpha_{i} \phi_{i}(T) \mathrm{d} T \\
& =\sum_{i=0}^{n} \alpha_{i} \gamma_{i}(X),
\end{aligned}
$$

where

$$
\gamma_{i}(X)=\int_{a}^{b} \int_{c}^{d} K(X, T) \phi_{i}(T) \mathrm{d} T .
$$

If we replace equations (16)-(18) into equation (1), the following equation will result:

$$
l(X)=\sum_{i=0}^{n} \alpha_{i} \phi_{i}(X)+A(X) \sum_{i=0}^{n} \alpha_{i} \phi_{i}(h(X))+\lambda \sum_{i=0}^{n} \alpha_{i} \gamma_{i}(X) .
$$

Now, put $X=x_{j}$ for $j=1,2, \ldots, n$. Then,

$$
l_{j}=\sum_{i=0}^{n} \alpha_{i} \phi_{i j}+A_{j} \alpha_{i} \bar{\phi}_{i j}+\lambda \sum_{i=0}^{n} \alpha_{i} \gamma_{i j}
$$

where $l_{j}=l\left(x_{j}\right), A_{j}=A\left(x_{j}\right)$.

$$
\begin{aligned}
& \bar{\phi}_{i j}=\phi_{i}\left(h\left(x_{j}\right)\right), \quad \bar{\phi}_{i j}=\phi_{i}\left(x_{j}\right), \\
& \gamma_{i j}=\gamma_{i}\left(x_{j}\right)=\int_{a}^{b} \int_{c}^{d} K\left(x_{j}, T\right) \phi_{i}(T) \mathrm{d} T .
\end{aligned}
$$

Matrix form for equation (21) is $\Delta \alpha=L$, in which $L=$ $\left(l_{1}, l_{2}, \ldots, l_{n}\right)^{T}$ and $\alpha=\left(\alpha_{1}, \alpha_{2}, \ldots, \alpha_{n}\right)^{T}$.

So, we have the following relations:

$$
\left[\begin{array}{cccc}
\Delta_{1,1} & \Delta_{1,2} & \ldots & \Delta_{1, n} \\
\Delta_{2,1} & \Delta_{2,2} & \ldots & \Delta_{2, n} \\
\vdots & \vdots & \ddots & \vdots \\
\Delta_{n, 1} & \Delta_{n, 2} & \ldots & \Delta_{n, n}
\end{array}\right]\left[\begin{array}{c}
\alpha_{1} \\
\alpha_{2} \\
\vdots \\
\alpha_{n}
\end{array}\right]=\left[\begin{array}{c}
l_{1} \\
l_{2} \\
\vdots \\
l_{n}
\end{array}\right],
$$

where

$$
\Delta_{i j}=\phi_{i j}+A_{j} \bar{\phi}_{i j}+\lambda \gamma_{i j} .
$$

Finally, Fredholm two-dimensional linear integral equation is approximated by the linear equation system of the $n$ variable; also, this method for functional equation (in mode $\lambda=0$ at equation (1)) is as follows:

$$
u(X)+A(X) u(h(X))=l(X) .
$$

It should be mentioned that the choice of the appropriate shape parameter in the RBF is critical to the numerical accuracy. Various studies $[25,26]$ have been performed to deal with this problem-dependent parameter in the RBF community. In the next section, the effectiveness of the present method along with its performance will be tested. In the next section, to show the efficiency and capability of the proposed approach, we examine it by providing examples. 


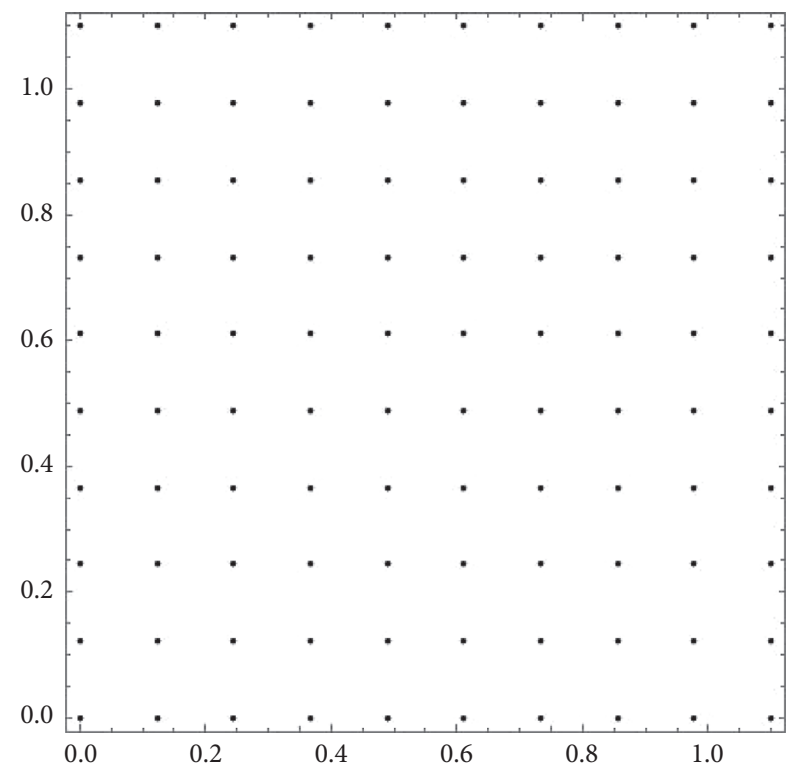

(a)

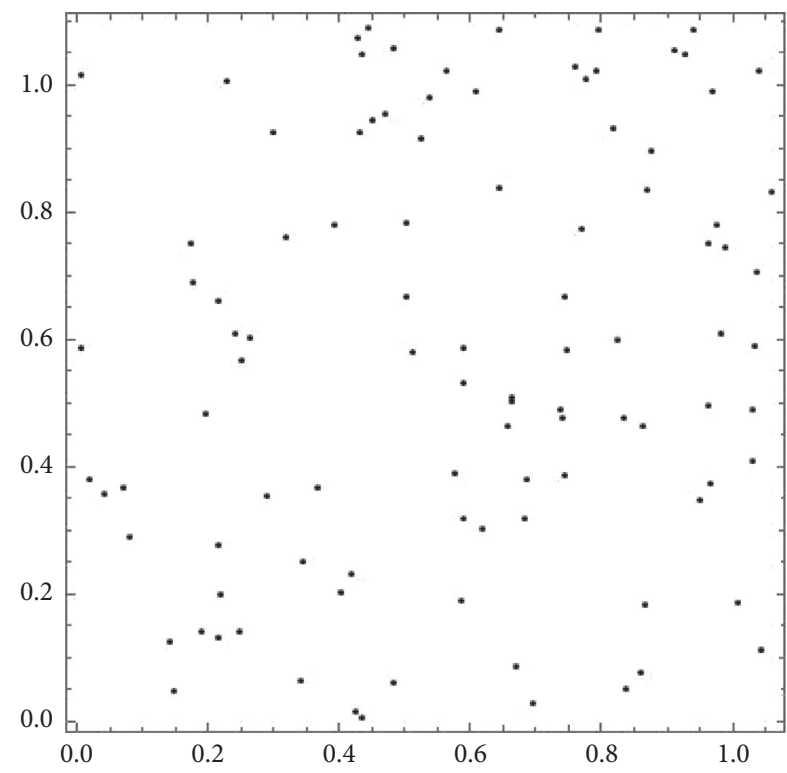

(b)

Figure 1: (a) The mesh points. (b) The meshless points. There are 100 points for interpolation.

\section{Numerical Examples}

The following examples have been presented to test efficiency of the suggested technique. Some numerical examples in this work are calculated by applying Mathematica software.

Example 1. Consider equation (1) which is defined in the Introduction section. We consider the points in both mesh and meshless case:

$$
u(X)+A(X) u(h(X))+\lambda \int_{a}^{b} \int_{c}^{d} K(X, T) u(T) \mathrm{d} T=l(X),
$$

where $X=\left(x_{1}, x_{2}\right), T=\left(t_{1}, t_{2}\right)$.

Also, $\quad A(X)=1, h(X)=X, \quad K(X, T)=e^{\left(x_{1}+x_{2}\right)-\left(t_{1}+t_{2}\right)}$, $l(X)=3.21 e^{\left(x_{1}+x_{2}\right)}$, and $a=0, b=1.1, c=0, d=1.1, \lambda=1$, where $u(X)=e^{\left(x_{1}+x_{2}\right)}$ is the exact solution.

So, integral equation is given in the following form:

$$
2 u(X)+\int_{0}^{1.1} \int_{0}^{1.1} e^{\left(x_{1}+x_{2}\right)-\left(t_{1}+t_{2}\right)} u(T) \mathrm{d} T=3.21 e^{x_{1}+x_{2}} .
$$

According to equation (24), we have the following relations:

$$
\Delta_{i j}=\phi_{i j}+A_{j} \bar{\phi}_{i j}+\lambda \gamma_{i j}
$$

where $\phi_{i j}=\phi_{i}\left(X_{j}\right)$ and $\bar{\phi}_{i j}=\phi_{i}\left(h\left(X_{j}\right)\right)$.

Now, can write

$$
\gamma_{i j}=\gamma_{i}\left(X_{j}\right)=\int_{0}^{1.1} \int_{0}^{1.1} e^{\left(x_{1}+x_{2}\right)-\left(t_{1}+t_{2}\right)} \phi_{i}(T) \mathrm{d} T .
$$

As mentioned in the previous section, $\alpha$ can be obtained from the $\Delta \alpha=L$ relation.
To do so, $u(X) \approx \sum_{i=0}^{100} \alpha_{i} \phi_{i}(X)$ has been defined. In all forms in the following, the mesh points on the left and the meshless points are located in the right side (Figures 1-4). Also, Table 2 provides the applications of RBF in solving Fredholm linear two-dimensional integral equation for 100 points in both mesh and meshless cases.

Example 2. In the introduction, we had equation (1):

$$
l(X)=u(X)+A(X) u(h(X))+\lambda \int_{a}^{b} \int_{c}^{d} K(X, T) u(T) \mathrm{d} T,
$$

where $X=\left(x_{1}, x_{2}\right), T=\left(t_{1}, t_{2}\right)$.

Let

$$
\begin{aligned}
A(X) & =e^{-\left(x_{1}+x_{2}\right)}, h(X)=\frac{X}{2}, \\
K(X, T) & =e^{\left(x_{1}+x_{2}\right)-\left(t_{1}+t_{2}\right)}, \\
l(X) & =e^{-\left(\left(x_{1} / 2\right)+\left(x_{2} / 2\right)\right)}+2.21 e^{\left(x_{1}+x_{2}\right),}, \\
a & =0, b=1.1, c=0, d=1.1, \lambda=1,
\end{aligned}
$$

where $u(X)=e^{\left(x_{1}+x_{2}\right)}$ is the exact solution.

We consider the points in both mesh and meshless cases, so we will have

$$
\begin{aligned}
& u(X)+e^{-\left(x_{1}+x_{2}\right)} u\left(\frac{X}{2}\right)+\int_{0}^{1.1} \int_{0}^{1.1} e^{\left(x_{1}+x_{2}\right)-\left(t_{1}+t_{2}\right)} u(T) \mathrm{d} T \\
& =e^{-\left(\left(x_{1} / 2\right)+\left(x_{2} / 2\right)\right)}+2.21 e^{\left(x_{1}+x_{2}\right)} .
\end{aligned}
$$



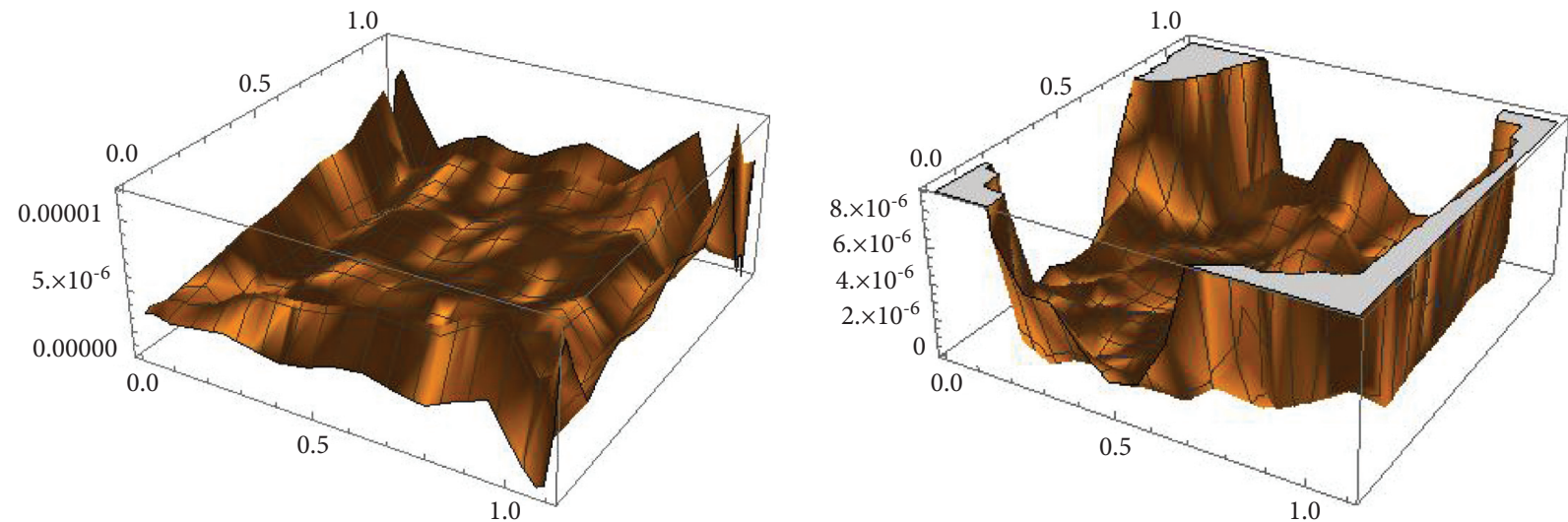

FIgURE 2: The plot 3D errors for both mesh and meshless points.
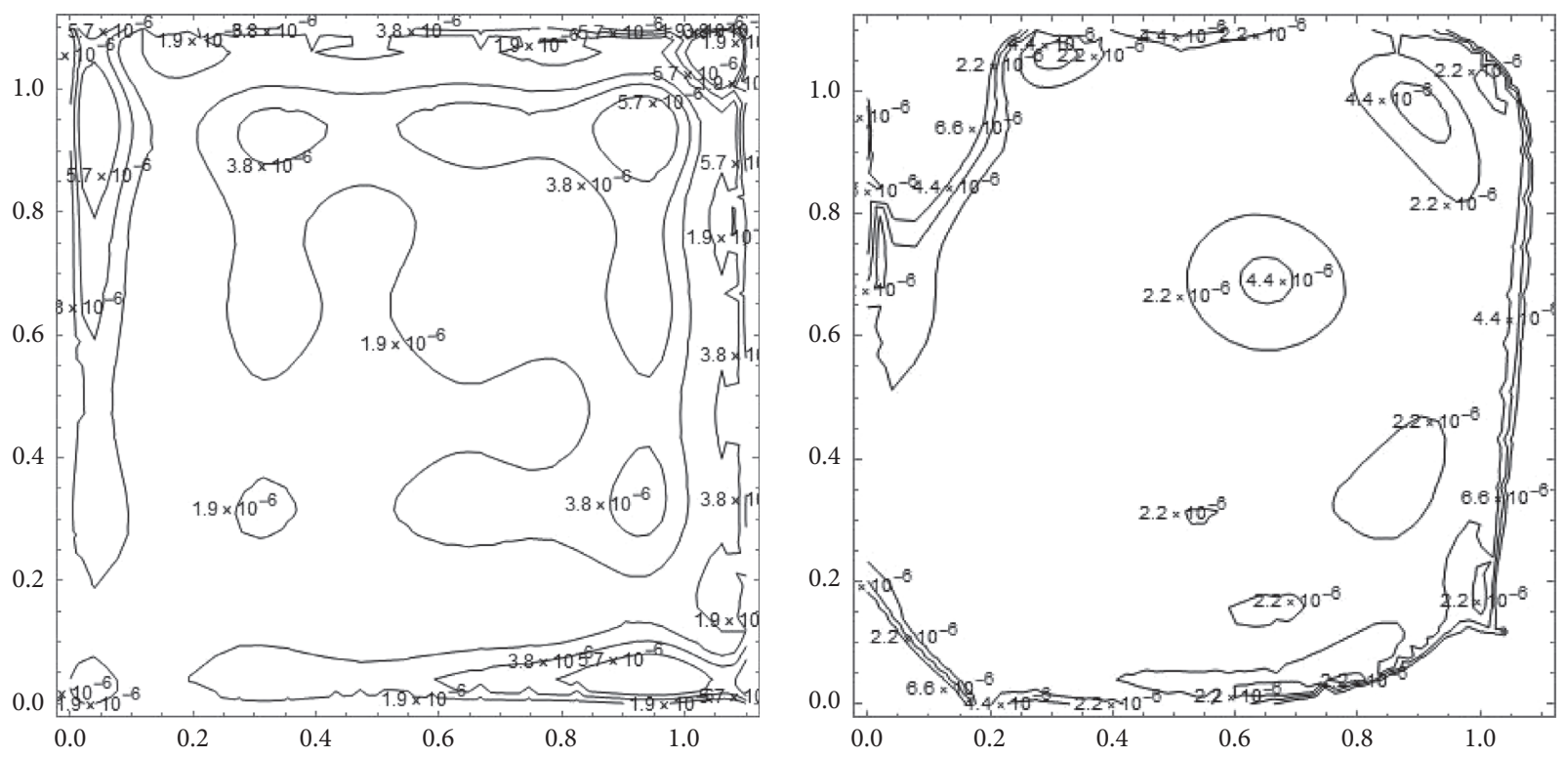

Figure 3: Contour plot errors in both mesh and meshless cases.
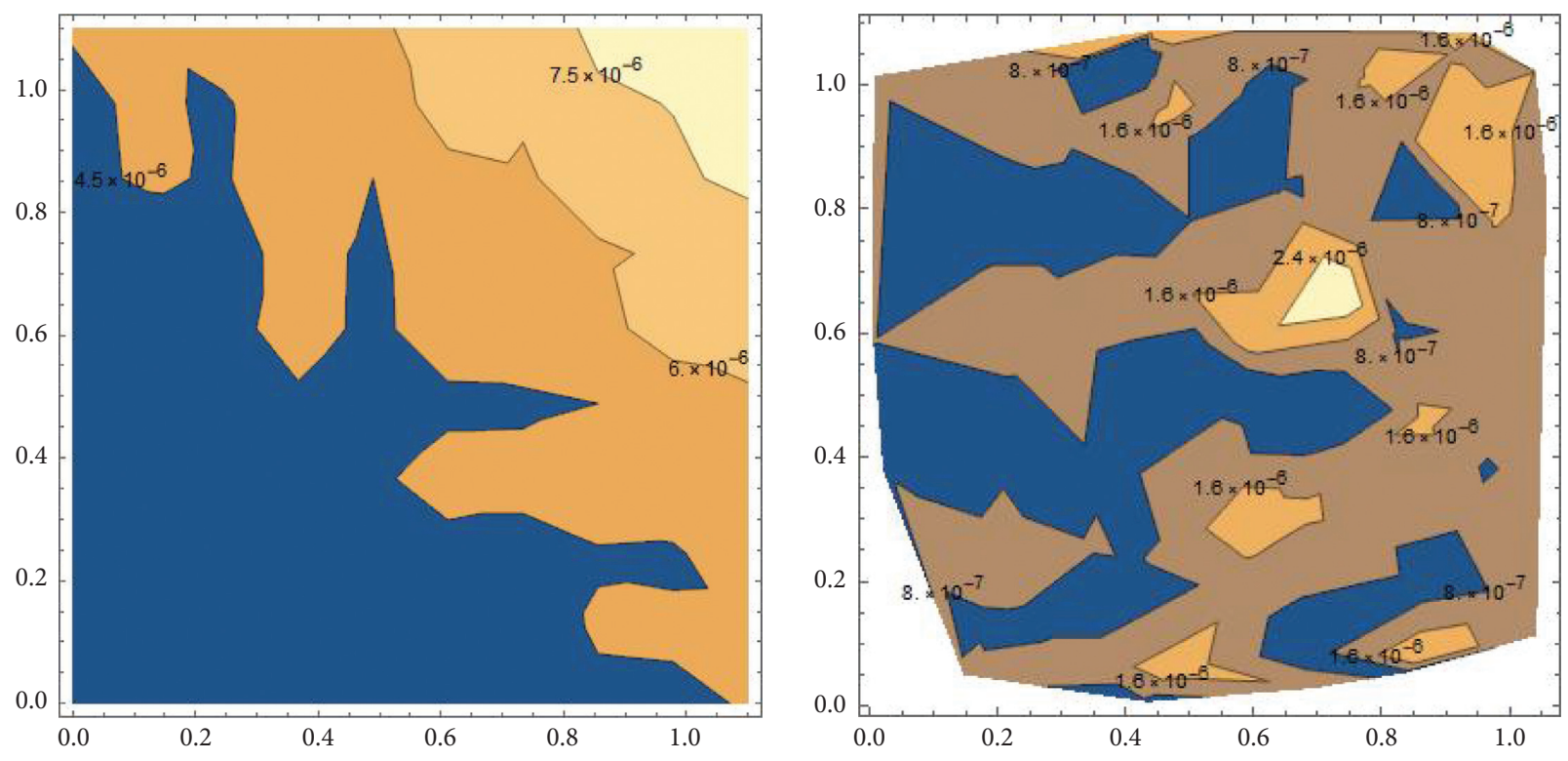

Figure 4: Contour plot errors for the same points. 
TABLE 2: Accuracy of RBF to Fredholm linear two-dimensional integral equation for mesh and meshless points.

\begin{tabular}{lcc}
\hline$N$ & $E_{\text {mesh points }}$ & $E_{\text {meshless points }}$ \\
\hline 9 & $9.25711 \times 10^{-2}$ & $7.33724 \times 10^{-2}$ \\
25 & $1.84293 \times 10^{-3}$ & $2.41075 \times 10^{-3}$ \\
49 & $3.22909 \times 10^{-5}$ & $1.41057 \times 10^{-5}$ \\
100 & $8.14192 \times 10^{-6}$ & $9.45367 \times 10^{-6}$ \\
\hline
\end{tabular}

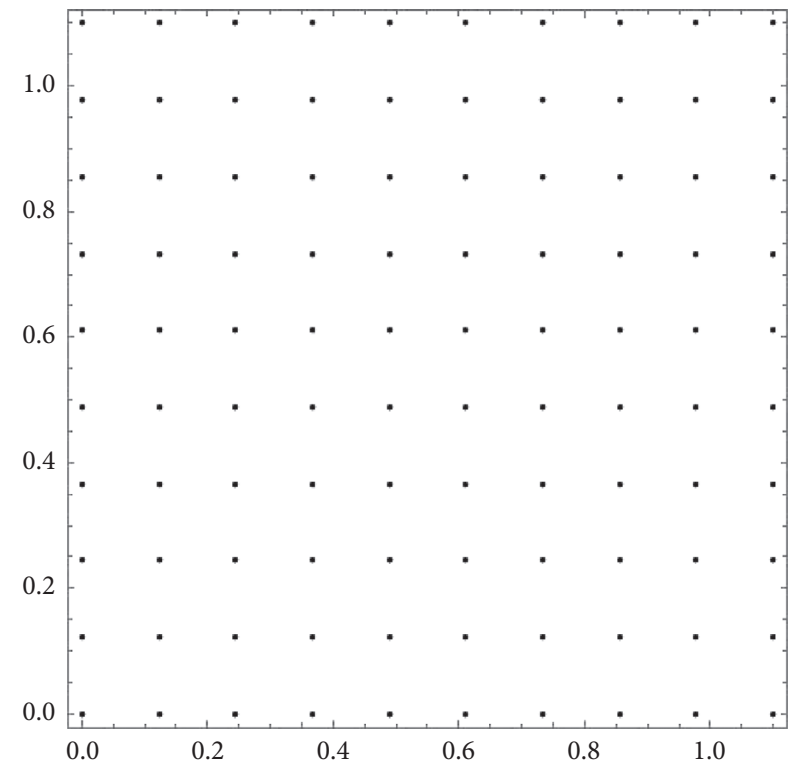

(a)

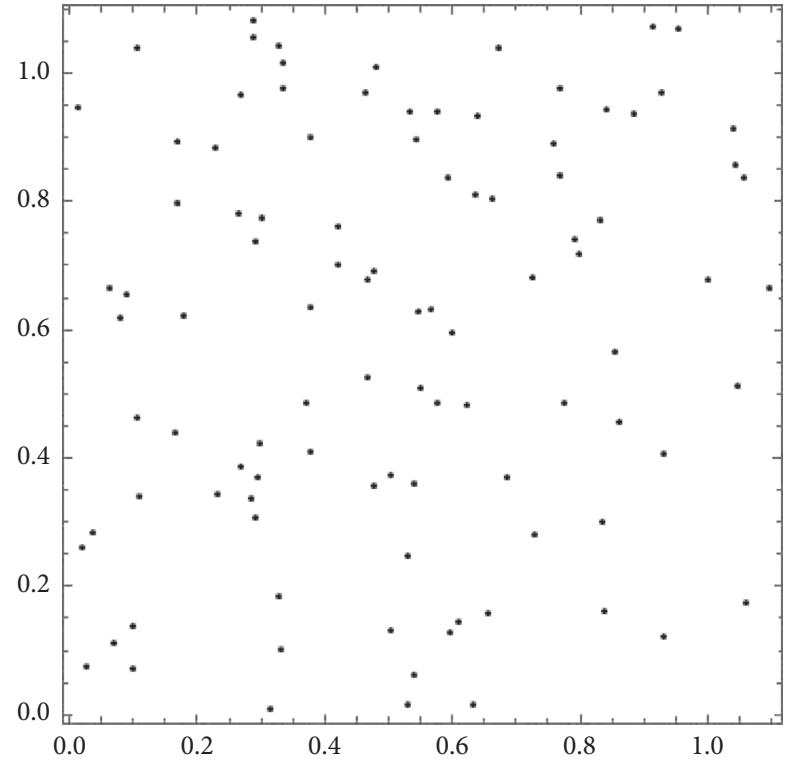

(b)

Figure 5: (a) The mesh points. (b) The meshless points. There are 100 points for interpolation.
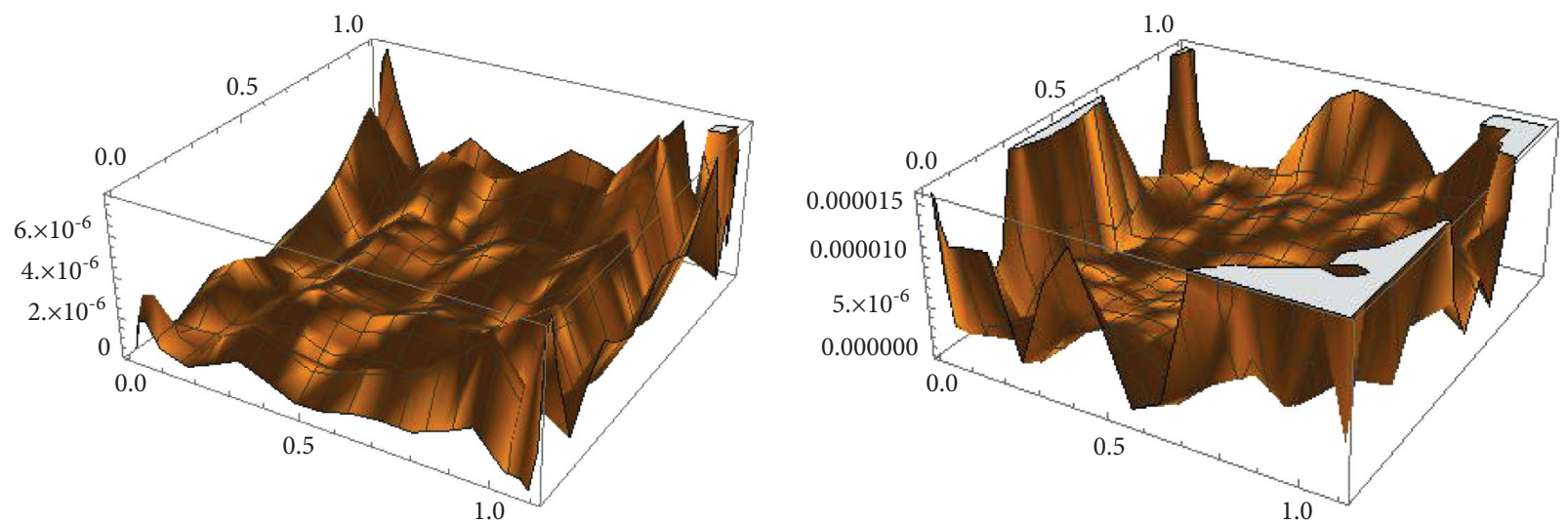

Figure 6: The plot 3D errors for both mesh and meshless points.

According to equation (24), the following relation is yielded:

$$
\Delta_{i j}=\phi_{i j}+A_{i j} \bar{\phi}_{i j}+\lambda \gamma_{i j}
$$

where $\phi_{i j}=\phi_{i}\left(X_{j}\right), \bar{\phi}_{i j}=\phi_{i}\left(h\left(X_{j}\right)\right)$.

So, we would have

$$
\gamma_{i j}=\gamma_{i}\left(X_{j}\right)=\int_{0}^{1.1} \int_{0}^{1.1} e^{\left(x_{1}+x_{2}\right)-\left(t_{1}+t_{2}\right)} \phi_{i}(T) \mathrm{d} T .
$$

Based on what we had before, $\Delta \alpha=L$, we will calculate the coefficient $\alpha$.

To do so, $u(X)=\sum_{i=0}^{100} \alpha_{i} \phi_{i}(X)$ is defined (Figures 5-8 and Table 3).

Example 3. Consider the following integral equation:

$$
l(X)=u(X)+A(X) u(h(X))+\lambda \int_{a}^{b} \int_{c}^{d} K(X, T) u(T) d T,
$$



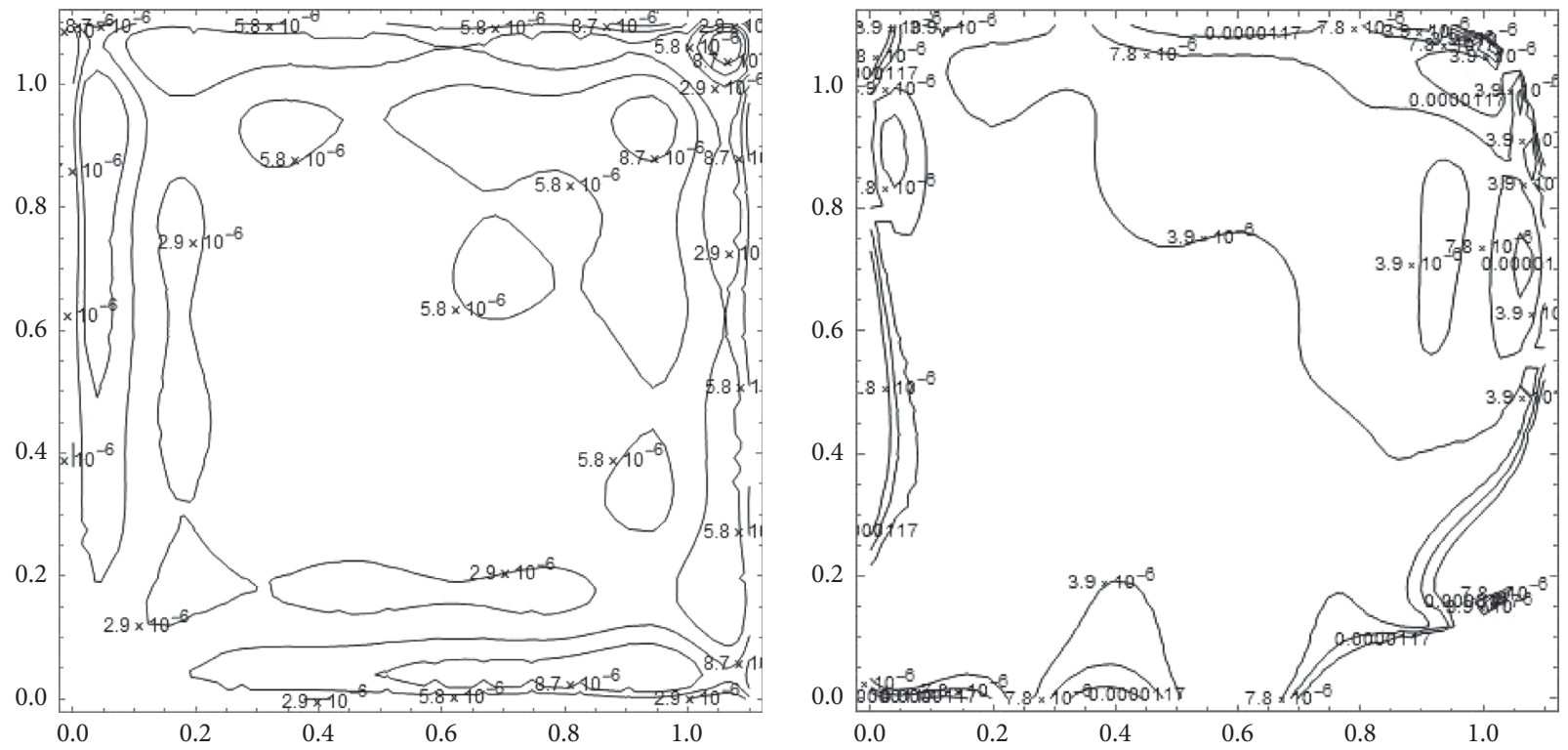

FIgURE 7: The Contour plot errors in both mesh and meshless cases.
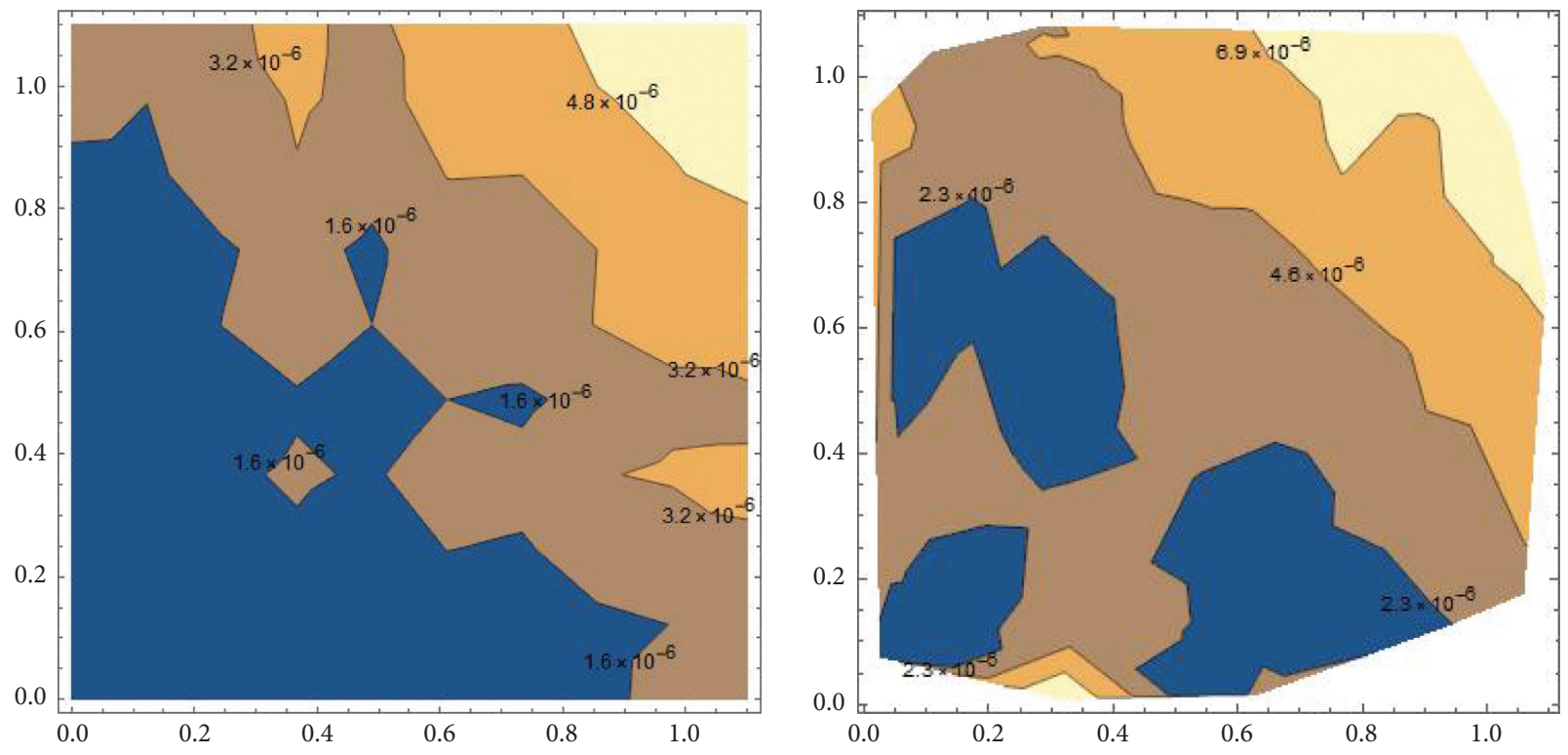

Figure 8: Contour plot errors for the same points.

TABle 3: Accuracy of RBF to Fredholm linear two-dimensional integral equation for mesh and meshless points.

\begin{tabular}{lcr}
\hline$N$ & $E_{\text {mesh points }}$ & $E_{\text {meshless points }}$ \\
\hline 9 & $5.85133 \times 10^{-2}$ & $3.85156 \times 10^{-2}$ \\
25 & $2.18032 \times 10^{-3}$ & $3.82658 \times 10^{-3}$ \\
49 & $6.55856 \times 10^{-5}$ & $7.16108 \times 10^{-5}$ \\
100 & $6.61838 \times 10^{-6}$ & $9.45367 \times 10^{-6}$ \\
\hline
\end{tabular}




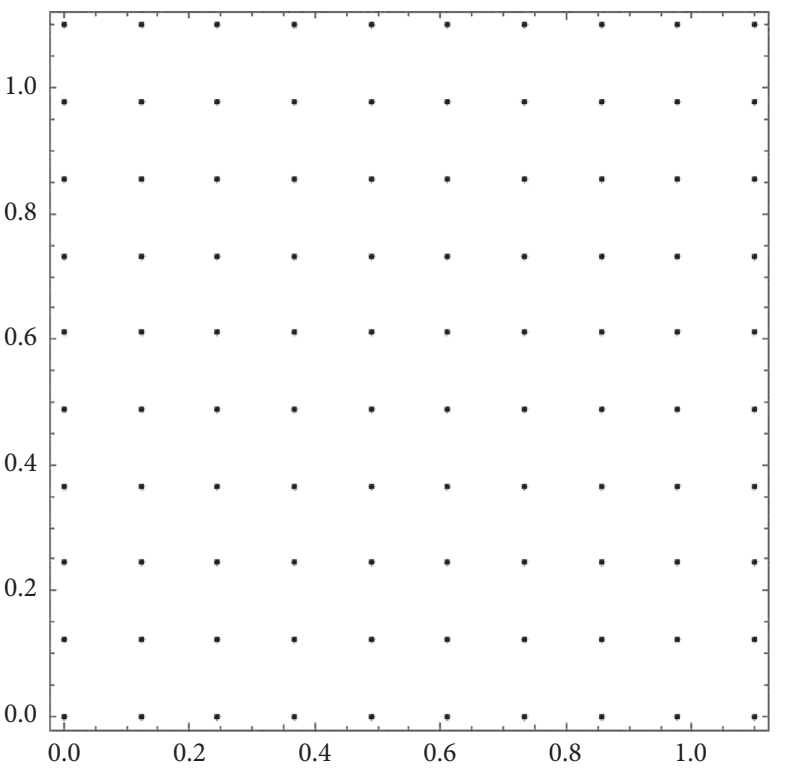

(a)

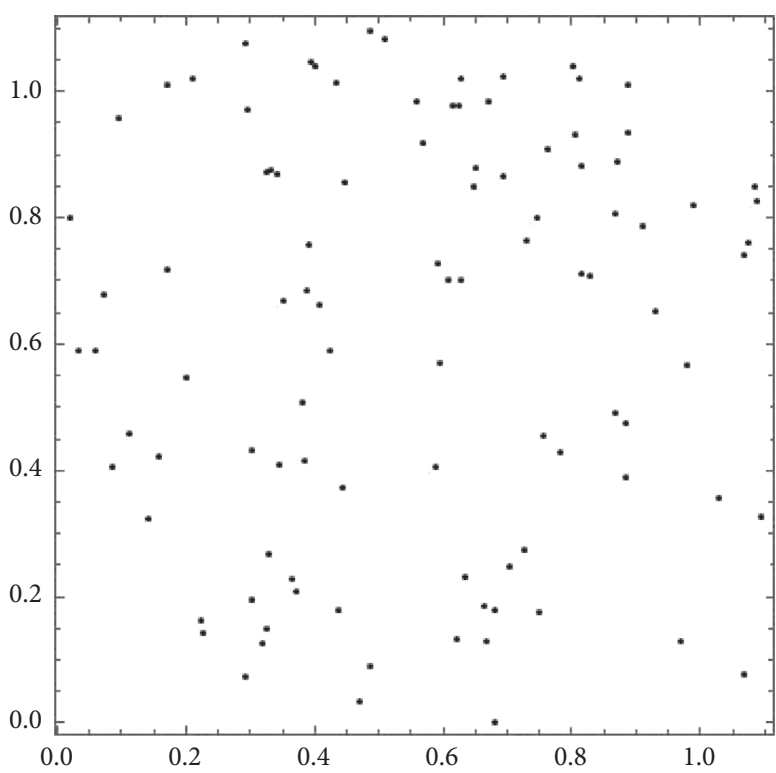

(b)

Figure 9: (a) The mesh points. (b) The meshless points. There are 100 points for interpolation.
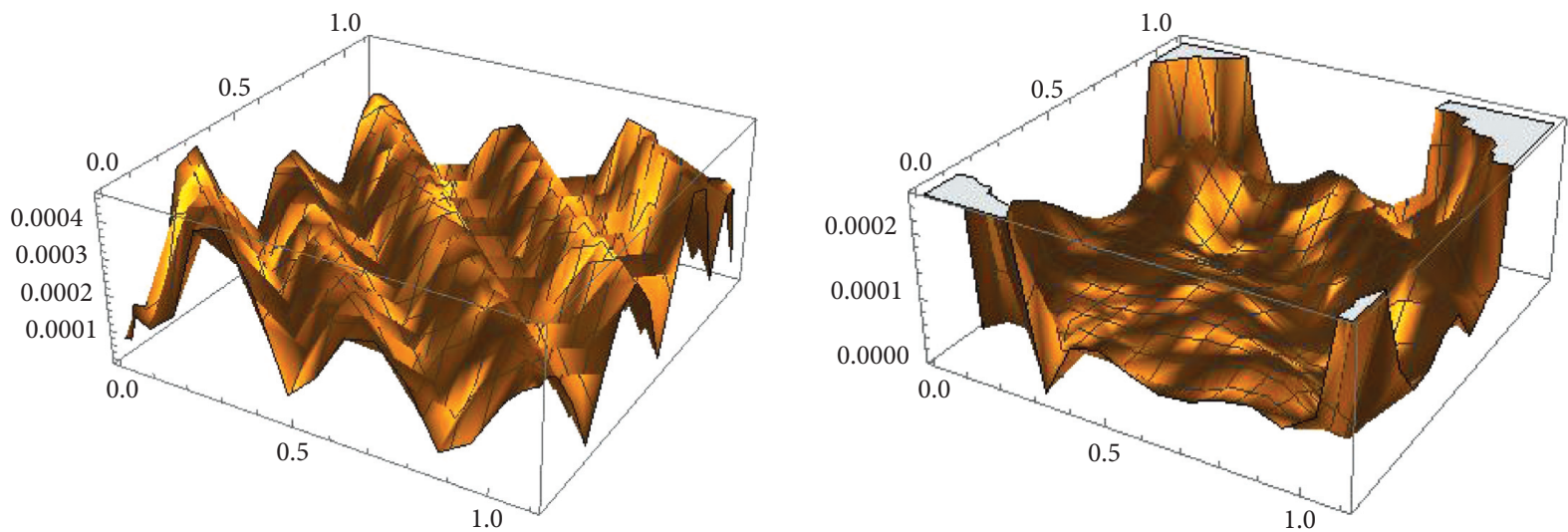

Figure 10: The plot 3D errors for both mesh and meshless points.
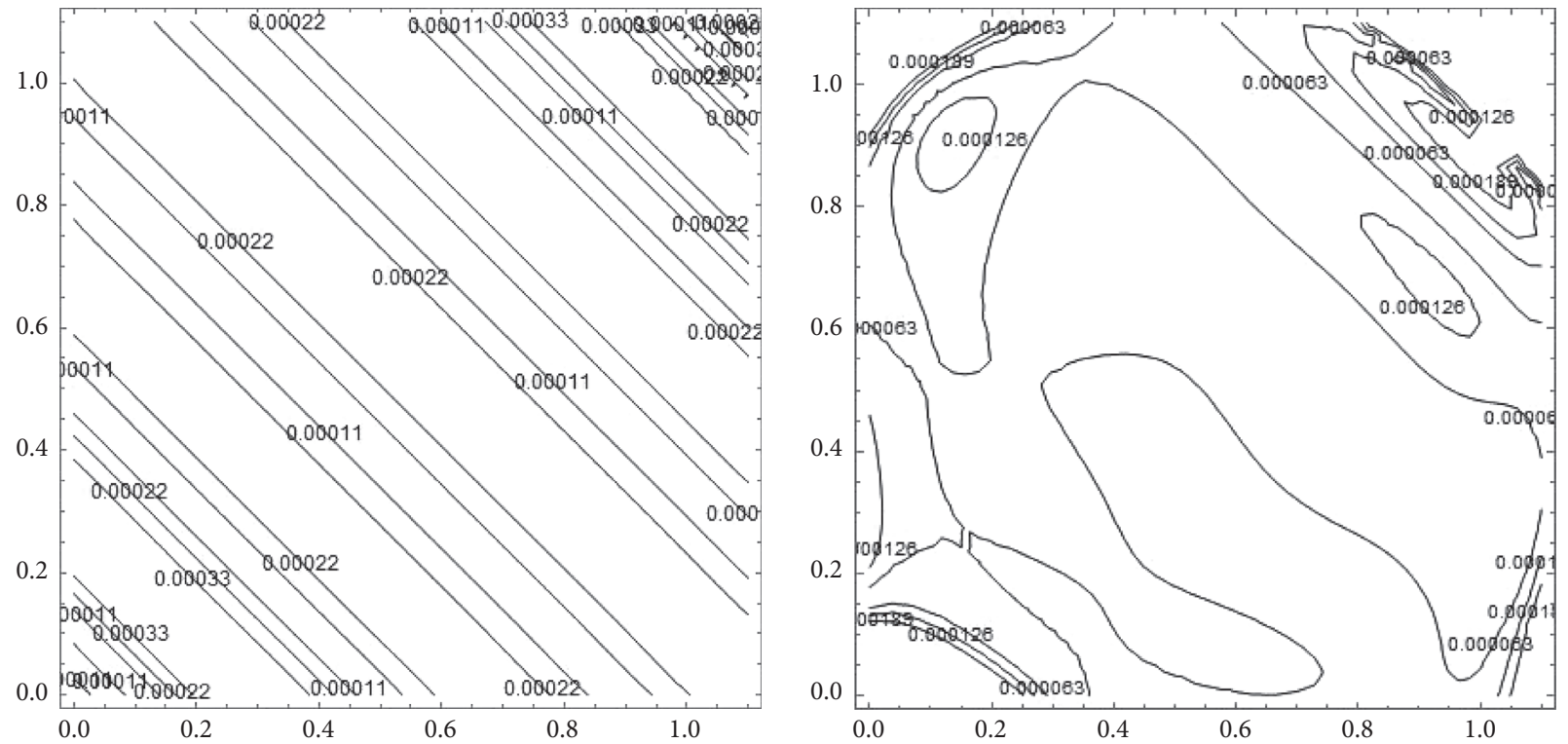

Figure 11: Contour plot errors in both mesh and meshless cases. 

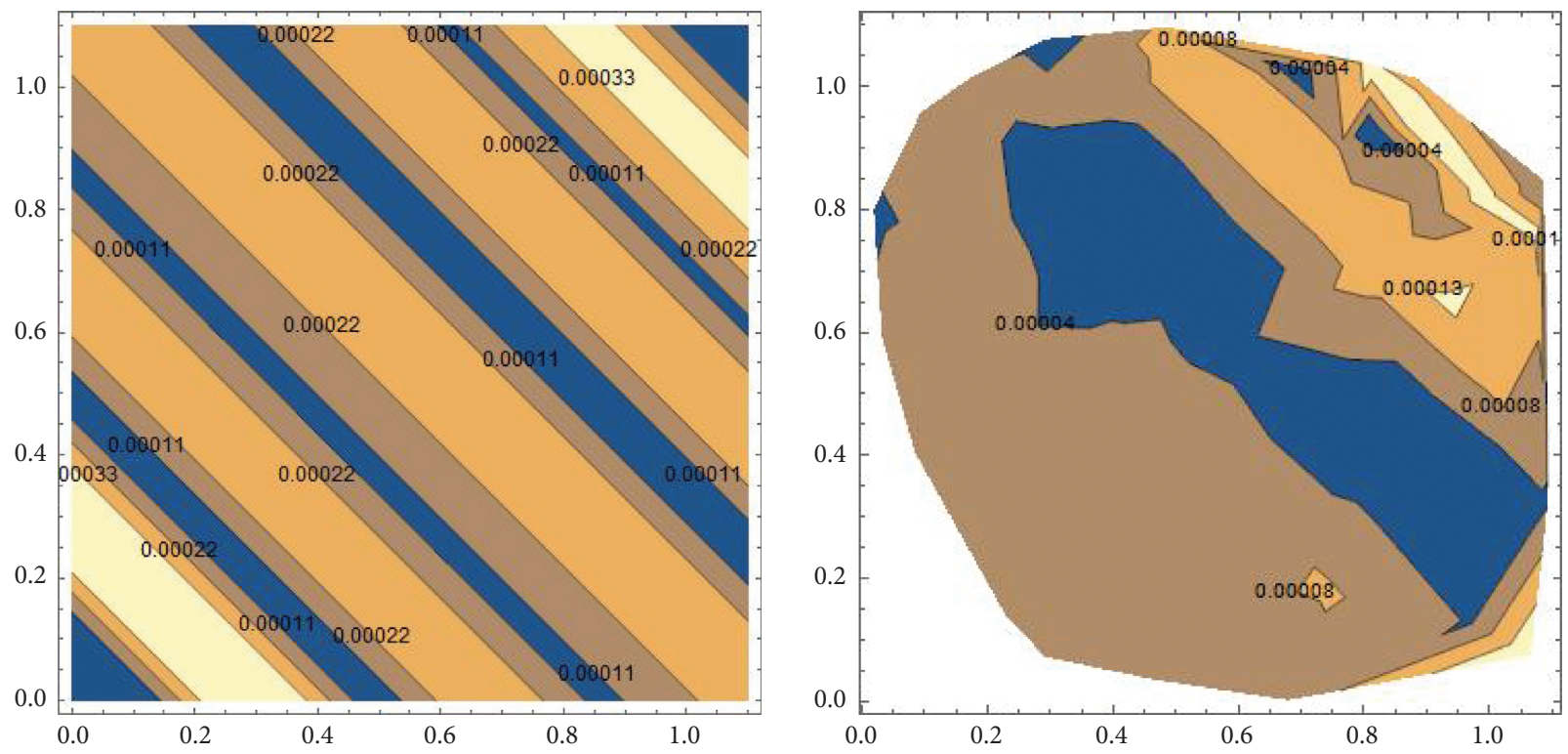

FIGURE 12: Contour plot errors for the same points.

TABLE 4: Accuracy of RBF to Fredholm linear two-dimensional integral equation for mesh and meshless points.

\begin{tabular}{lcr}
\hline$N$ & $E_{\text {mesh points }}$ & $E_{\text {meshless points }}$ \\
\hline 9 & $9.44726 \times 10^{-3}$ & $4.163663 \times 10^{-2}$ \\
25 & $2.65143 \times 10^{-4}$ & $3.17089 \times 10^{-3}$ \\
49 & $8.02597 \times 10^{-4}$ & $5.13247 \times 10^{-3}$ \\
100 & $4.50859 \times 10^{-4}$ & $1.78766 \times 10^{-4}$ \\
\hline
\end{tabular}

where $X=\left(x_{1}, x_{2}\right), T=\left(t_{1}, t_{2}\right)$.

Let

$$
\begin{aligned}
A(X)= & 1, h(X)=X, \\
a= & 0, b=1.1, c=0, d=1.1, \\
K(X, T)= & \cos \left[\left(x_{1}+x_{2}\right)-\left(t_{1}+t_{2}\right)\right], \\
l(X)= & -0.125 \sin \left(-x_{1}-x_{2}\right)+0.25 \sin \left[2.2-x_{1}-x_{2}\right] \\
& -0.125 \sin \left[4.4-x_{1}-x_{2}\right]+2.605 \sin \left(x_{1}+x_{2}\right),
\end{aligned}
$$

where $u(X)=\sin \left(x_{1}+x_{2}\right)$ is the exact solution. We consider the points in both mesh and meshless cases, so we will have

$$
\begin{gathered}
2 u(X)+\int_{0}^{1.1} \int_{0}^{1.1} \cos \left[\left(x_{1}+x_{2}\right)-\left(t_{1}+t_{2}\right)\right] u(T) \mathrm{d} T \\
=-0.125 \sin \left(-x_{1}-x_{2}\right)+0.25 \sin \left[2.2-x_{1}-x_{2}\right] \\
\quad-0.125 \sin \left[4.4-x_{1}-x_{2}\right]+2.605 \sin \left(x_{1}+x_{2}\right) .
\end{gathered}
$$

According to equation (24) we have the following relation:

$$
\Delta_{i j}=\phi_{i j}+A_{i j} \bar{\phi}_{i j}+\lambda \gamma_{i j}
$$

where $l_{j}=l\left(x_{j}\right), A_{j}=A\left(x_{j}\right), \phi_{i j}=\phi_{i}\left(x_{j}\right)$, and $\bar{\phi}_{i j}=\phi_{i}$ $\left(h\left(x_{j}\right)\right)$. So, we would have

$$
\gamma_{i j}=\gamma_{i}\left(x_{j}\right)=\int_{0}^{1.1} \int_{0}^{1.1} \cos \left[\left(x_{1}+x_{2}\right)-\left(t_{1}+t_{2}\right)\right] \phi_{i}(T) \mathrm{d} T
$$

According to what we had before, $\Delta \alpha=L$, we will calculate the coefficient $\alpha$.

To this aim, $u(X)=\sum_{i=0}^{100} \alpha_{i} \phi_{i}(x)$ is defined (Figures 9-12 and Table 4).

\section{Conclusion}

The RBF method was proposed for solving the Fredholm functional two-dimensional linear integral equations. These types of equations are very significant, and sometimes, detecting their analytic solutions is so difficult. Thus, using a useful numerical method for these types of equations is a topic of interest for researchers. At the end, the accuracy and applicability of the proposed method are shown through some numerical applications. The method is performed on three test problems, and simulation results show that the numerical method is appropriate for obtaining the approximate solutions of our models. Also, this method has higher accuracy and fewer calculations. The RBF method can also be used for higher dimensional and both linear and nonlinear integral equations. 


\section{Data Availability}

No data were used to support this study.

\section{Conflicts of Interest}

The authors declare that they have no conflicts of interest.

\section{References}

[1] X.-M. Gu and S.-L. Wu, "A parallel-in-time iterative algorithm for volterra partial integro-differential problems with weakly singular kernel," Journal of Computational Physics, vol. 417, Article ID 109576, 2020.

[2] Y. L. Zhao, X. M. Gu, and A. Ostermann, "A preconditioning technique for an all-at-once system from volterra subdiffusion equations with graded time steps," Journal of Scientific Computing, vol. 88, no. 11, pp. 1-22, 2021.

[3] I. G. Graham, "Collocation methods for two dimensional weakly singular integral equations," The Journal of the Australian Mathematical Society: Series B. Applied Mathematics, vol. 22, no. 4, pp. 456-473, 1981.

[4] S. McKee, T. Tang, and T. Diogo, "An euler-type method for two-dimensional volterra integral equations of the first kind," IMA Journal of Numerical Analysis, vol. 20, no. 3, pp. 423440, 2000.

[5] M. T. Rashed, "Numerical solution of functional differential, integral and integro-differential equations," Applied Mathematics and Computation, vol. 156, no. 2, pp. 485-492, 2004.

[6] C. Franke and R. Schaback, "Solving partial differential equations by collocation using radial basis functions," Applied Mathematics and Computation, vol. 93, no. 1, pp. 73-82, 1998.

[7] W. Chen, Z. J. Fu, and C. S. Chen, Recent Advances in Radial Basis Function Collocation Methods, Springer, Heidelberg, Germany, 2014

[8] L. Derog, Ch. Tsu-Shuan, and Z. Yi, "A constructive algorithm for feed forward neural networks with incremental training," IEEE Transactions on Circuits and Systems, vol. 49, pp. 1876-1879, 2002.

[9] C. de Boor, "Multivariate piecewise polynomials," Acta Numerica, vol. 2, pp. 65-109, 1993.

[10] R. L. Hardy, "Multiquadric equations of topography and other irregular surfaces," Journal of Geophysical Research, vol. 76, no. 8, pp. 1905-1915, 1971.

[11] R. L. Hardy, "Theory and applications of the multiquadricbiharmonic method 20 years of discovery 1968-1988," Computers \& Mathematics with Applications, vol. 19, no. 8-9, pp. 163-208, 1990.

[12] R. Franke, "Scattered data interpolation: tests of some methods," Mathematics of Computation, vol. 38, no. 157, p. $181,1982$.

[13] C. A. Micchelli, "Interpolation of scattered data: distance matrices and conditionally positive definite functions," Constructive Approximation, vol. 2, no. 1, pp. 11-22, 1986.

[14] M. D. Buhmann and C. A. Micchelli, "Multiquadric interpolation improved advanced in the theory and applications of radial basis functions," Computers \& Mathematics with Applications, vol. 43, no. 12, pp. 21-25, 1995.

[15] E. J. Kansa, "Multiquadrices-a scattered data approximation scheme with applications to computational fluid-dynamics, I: surface approximations and partial derivative estimates," Computers \& Mathematics with Applications, vol. 19, no. 819, pp. 127-145, 1990.
[16] E. J. Kansa, "Multiquadrics-a scattered data approximation scheme with applications to computational fluid-dynamics-II solutions to parabolic, hyperbolic and elliptic partial differential equations," Computers \& Mathematics with Applications, vol. 19, no. 8-9, pp. 147-161, 1990.

[17] R. Firouzdor, S. S. Asari, and M. Amirfakhrian, "Application of radial basis function to approximate functional integral equations," Journal of Interpolation and Approximation in Scientific Computing, vol. 2016, no. 2, pp. 77-86, 2016.

[18] M. Tatari, B. Sepehrian, and M. Alibakhshi, "New implementation of radial basis functions for solving Burgers-Fisher equation," Numerical Methods for Partial Differential Equations, vol. 28, no. 1, pp. 248-262, 2012.

[19] W. Hu, Adaptive Multiscale Meshfree Method for Sloving the Schrodinger Equation in Quantum Mechanics, University of California, Los Angeles, CA, USA, 2011.

[20] J. Biazar, M. Gholami Porshokouhi, B. Ghanbari, and M. Gholami Porshokouhi, "Numerical solution of functional integral equations by the variational iteration method," Journal of Computational and Applied Mathematics, vol. 235, no. 8, pp. 2581-2585, 2011.

[21] A. Alipanah and S. Esmaeili, "Numerical solution of the twodimensional Fredholm integral equations using Gaussian radial basis function," Journal of Computational and Applied Mathematics, vol. 235, no. 18, pp. 5342-5347, 2011.

[22] S. S. Asari, M. Amirfakhrian, and S. Chakraverty, "Application of radial basis functions in solving fuzzy integral equations," Neural Computing \& Applications, vol. 31, no. 10, pp. 6373-6381, 2019.

[23] H. Wendland, Scattered Data Aproximation, Cambridge University Press, Cambridge, UK, 2005.

[24] Z. E. Majouti, R. E. Jid, and A. Hajjaj, "Solving two-dimensional linear and nonlinear mixed integral equations using moving least squares and modified moving least squares methods," International Journal of Applied Mathematics, vol. 51, no. 1, 2021.

[25] Z.-J. Fu, J. Zhang, P.-W. Li, and J.-H. Zheng, "A semi-Lagrangian meshless framework for numerical solutions of twodimensional sloshing phenomenon," Engineering Analysis with Boundary Elements, vol. 112, pp. 58-67, 2020.

[26] G. E. Fasshauer and J. G. Zhang, "On choosing "optimal" shape parameters for RBF approximation," Numerical Algorithms, vol. 45, no. 1, pp. 345-368, 2007. 